\title{
Quantum mechanical path integrals in curved spaces and the type-A trace anomaly
}

\author{
Fiorenzo Bastianelli, ${ }^{a, b}$ Olindo Corradini ${ }^{c, b}$ and Edoardo Vassura ${ }^{a, b}$ \\ ${ }^{a}$ Dipartimento di Fisica ed Astronomia, Università di Bologna, \\ via Irnerio 46, I-40126 Bologna, Italy \\ ${ }^{b}$ INFN, Sezione di Bologna, \\ via Irnerio 46, I-40126 Bologna, Italy \\ ${ }^{c}$ Dipartimento di Scienze Fisiche, Informatiche e Matematiche, \\ Università di Modena e Reggio Emilia, \\ Via Campi 213/A, I-41125 Modena, Italy \\ E-mail: fiorenzo.bastianelli@bo.infn.it, olindo.corradini@unimore.it, \\ edoardo.vassura@studio.unibo.it
}

ABStRact: Path integrals for particles in curved spaces can be used to compute trace anomalies in quantum field theories, and more generally to study properties of quantum fields coupled to gravity in first quantization. While their construction in arbitrary coordinates is well understood, and known to require the use of a regularization scheme, in this article we take up an old proposal of constructing the path integral by using Riemann normal coordinates. The method assumes that curvature effects are taken care of by a scalar effective potential, so that the particle lagrangian is reduced to that of a linear sigma model interacting with the effective potential. After fixing the correct effective potential, we test the construction on spaces of maximal symmetry and use it to compute heat kernel coefficients and type-A trace anomalies for a scalar field in arbitrary dimensions up to $d=12$. The results agree with expected ones, which are reproduced with great efficiency and extended to higher orders. We prove explicitly the validity of the simplified path integral on maximally symmetric spaces. This simplified path integral might be of further use in worldline applications, though its application on spaces of arbitrary geometry remains unclear.

Keywords: Anomalies in Field and String Theories, Sigma Models

ArXIV EPRINT: 1702.04247 


\section{Contents}

1 Introduction 1

2 Particle in curved space $\quad 2$

$\begin{array}{lll}3 & \text { A linear sigma model } & 6\end{array}$

$\begin{array}{lll}4 & \text { Path integral on maximally symmetric spaces } & 7\end{array}$

5 Type-A trace anomaly of a scalar field 10

6 Conclusions 12

$\begin{array}{ll}\text { A A simple proof in maximally symmetric spaces } & 13\end{array}$

$\begin{array}{ll}\text { B Computational details } & 13\end{array}$

\section{Introduction}

The path integral formulation of quantum mechanics [1] carries a certain amount of subtleties when applied to particles moving in a curved background. These subtleties are the analogue of the ordering ambiguities of canonical quantization, and can be addressed by specifying a regularization scheme needed to make sense of the path integral, at least perturbatively. The action of a nonrelativistic particle takes the form of a nonlinear sigma model in one dimension, and as such it identifies a super-renormalizable one-dimensional quantum field theory. It can be treated by choosing a regularization scheme supplemented by corresponding counterterms, the latter being needed to match the renomalization conditions, i.e. to fix uniquely the theory under study.

While several regularization schemes have been worked out and tested, see [2], in this article we take up an old proposal, put forward by Guven in [3], of constructing the path integral in curved spaces by making use of Riemann normal coordinates. It assumes that in such a coordinate system an auxiliary flat metric can be used in the kinetic term, while a suitable effective potential is supposed to reproduce the effects of the curved space. This construction transforms the model into a linear sigma model. The simplifications expected in having a linear sigma model, rather then a nonlinear one, are rather appealing, and motivated us to investigate the issue further. Indeed, a simplified path integral might be more efficient for perturbative calculations, making worldline applications easier. We shall apply and test the method on spaces of maximal symmetry (e.g. spheres) by perturbatively computing the partition function, and check if it reproduces known results. This happens with a dramatic gain in efficiency. We recall that the partition function on spheres can be used 
as generating function for the type-A trace anomalies of a scalar field in arbitrary $d$ dimensions. The evaluation of trace anomalies is a typical worldline calculation, performed in [4] up to $d=6$ by using the nonlinear sigma model. The linear sigma model allows to reproduce those results and to push the perturbative order much further. We use it to scan dimensions up to $d=12$, though one could go higher if needed. Our conclusion is that the method is viable on spaces of maximal symmetry, and indeed we provide an explicit proof of its validity. However, an extension to generic curved spaces is not warranted, as we shall discuss later on.

We structure our paper as follows. We first review the path integral construction in arbitrary coordinates, to put the new method in the right perspective. The action in arbitrary coordinates is that of a nonlinear sigma model, and we seize the opportunity to comment on its use in worldline applications. In section 3 we review the proposal of ref. [3], and point out that the identification of the effective potential reported in that reference is incorrect (though it could be a misprint). More importantly, we stress that the proof of why the effective potential should work is not given in ref. [3], nor is it contained in the cited references. In some of those references [5, 6], see also [7], we have found arguments why the assumption of an effective potential might work perturbatively, at least up to few perturbative orders. Those arguments use the Lorentz symmetry of flat space recursively, and do not seem to apply on generic curved spaces. Thus in section 4 we restrict ourselves to spaces of maximal symmetry, where those arguments might have a better chance of working. We test the method with the correct effective potential by computing perturbatively the partition function. We find indeed that it reproduces more efficiently known results. Moreover it permits to push the calculations to higher perturbative orders. In section 5 we use the partition function to extract the type-A trace anomalies for a scalar field in arbitrary $d$ dimensions up to $d=12$. This produces further checks on the path integral results. Conforted by this success, we are led to provide an explicit proof of the validity of the simplified path integral on maximally symmetric spaces, which is presented in appendix A, while appendix B is left for details on our linear sigma-model worldline calculations.

\section{Particle in curved space}

The lagrangian of a nonrelativistic particle of unit mass in a curved $d$-dimensional space contains just the kinetic term

$$
L(x, \dot{x})=\frac{1}{2} g_{i j}(x) \dot{x}^{i} \dot{x}^{j}
$$

where $g_{i j}(x)$ is the metric in an arbitrary coordinate system. It is the action of a nonlinear sigma model in one dimension, and the corresponding equations of motion are the geodesic equations written in terms of the affine parameter $t$, the time used in the definition of the velocity $\dot{x}^{i}=\frac{d x^{i}}{d t}$. The corresponding hamiltonian reads

$$
H(x, p)=\frac{1}{2} g^{i j}(x) p_{i} p_{j}
$$

where $p_{i}$ are the momenta conjugated to $x^{i}$. Upon canonical quantization it carries ordering ambiguities, which consist in terms containing one or two derivatives acting on the 
metric. ${ }^{1}$ These ambiguities are greatly reduced by requiring background general coordinate invariance. Since the only tensor that can be constructed with one and two derivatives on the metric is the curvature tensor, the most general diffeomorphism invariant quantum hamiltonian takes the form

$$
\hat{H}=-\frac{1}{2} \nabla^{2}+\frac{\xi}{2} R
$$

where $\nabla^{2}$ is the covariant laplacian acting on scalar wave functions, and $\xi$ is an arbitrary coupling to the scalar curvature $R$ (defined to be positive on a sphere) that parametrizes remaining ordering ambiguities. The value $\xi=0$ defines the minimal coupling, while the value $\xi=\frac{d-2}{4(d-1)}$ is the conformally invariant coupling in $d$ dimensions.

For definiteness let us review the theory with the minimal coupling $\xi=0$. Other values can be obtained by simply adding a scalar potential $V=\frac{\xi}{2} R$. The transition amplitude in euclidean time $\beta$ (the heat kernel)

$$
K\left(x, x^{\prime} ; \beta\right) \equiv\left\langle x\left|e^{-\beta \hat{H}(\hat{x}, \hat{p})}\right| x^{\prime}\right\rangle
$$

is defined with the covariant hamiltonian ${ }^{2}$

$$
\hat{H}(\hat{x}, \hat{p})=\frac{1}{2} g^{-\frac{1}{4}}(\hat{x}) \hat{p}_{i} g^{i j}(\hat{x}) g^{\frac{1}{2}}(\hat{x}) \hat{p}_{j} g^{-\frac{1}{4}}(\hat{x}) .
$$

It solves the Schroedinger equation in euclidean time (heat equation)

$$
-\frac{\partial}{\partial \beta} K\left(x, x^{\prime} ; \beta\right)=-\frac{1}{2} \nabla_{x}^{2} K\left(x, x^{\prime} ; \beta\right)
$$

and satisfies the boundary condition at $\beta \rightarrow 0$

$$
K\left(x, x^{\prime} ; 0\right)=\frac{\delta^{(d)}\left(x-x^{\prime}\right)}{\sqrt{g(x)}} .
$$

In eq. (2.6) $\nabla_{x}^{2}$ indicates the covariant scalar laplacian acting on coordinates $x$.

The transition amplitude $K\left(x, x^{\prime} ; \beta\right)$ can be given a path integral representation. Using a Weyl reordering of the quantum Hamiltonian $\hat{H}(\hat{x}, \hat{p})$ allows to derive a discretized phasespace path integral containing the classical phase-space action suitably discretized by the midpoint rule [9]. The action acquires a finite counterterm $V_{\mathrm{TS}}$ of quantum origin, arising form the Weyl reordering of the specific hamiltonian in eq. (2.5), originally performed in [10] (the subscript TS reminds of the time slicing discretization of the time variable). The perturbative evaluation of the phase space path integral can be performed directly in the continuum limit [11]

$$
\int D x D p e^{-S[x, p]}
$$

\footnotetext{
${ }^{1}$ In the coordinate representation the hermitian momentum acting on a scalar wave function takes the form $p_{i}=-i g^{-\frac{1}{4}} \partial_{i} g^{\frac{1}{4}}$. Further details may be found in the book [2], or in the classic paper [8].

${ }^{2}$ We choose position eigenstates normalized as scalars: $\hat{x}^{i}|x\rangle=x^{i}|x\rangle,\left\langle x \mid x^{\prime}\right\rangle=\frac{\delta^{(d)}\left(x-x^{\prime}\right)}{\sqrt{g(x)}}, \nVdash=$ $\int d^{d} x \sqrt{g(x)}|x\rangle\langle x|$, so that the amplitude $K\left(x, x^{\prime} ; \beta\right)$ is a biscalar.
} 
with the phase-space euclidean action taking the form

$$
\begin{aligned}
S[x, p] & =\int_{0}^{\beta} d t\left(-i p_{i} \dot{x}^{i}+H(x, p)\right) \\
H(x, p) & =\frac{1}{2} g^{i j}(x) p_{i} p_{j}+V_{\mathrm{TS}}(x) \\
V_{\mathrm{TS}}(x) & =-\frac{1}{8} R(x)+\frac{1}{8} g^{i j}(x) \Gamma_{i k}^{l}(x) \Gamma_{j l}^{k}(x) .
\end{aligned}
$$

To generate the amplitude $K\left(x, x^{\prime} ; \beta\right)$ the paths $x(t)$ must satisfy the boundary conditions $x(0)=x^{\prime}$ and $x(\beta)=x$, while the paths $p(t)$ are unconstrained. We recall that perturbative corrections are finite in phase space. The presence of the noncovariant part of the counterterm $V_{\mathrm{TS}}$ corrects the noncovariance of the midpoint discretization, and it makes sure that the final result is covariant. These noncovariant counterterms were also derived in [12] (and reviewed in the book [13]) by considering point transformations (i.e. arbitrary changes of coordinates) in flat space.

The definition of the corresponding path integral in configuration space encounters more subtle problems. The classical action takes the form of a nonlinear sigma model in one dimension

$$
S[x]=\int_{0}^{\beta} d t \frac{1}{2} g_{i j}(x) \dot{x}^{i} \dot{x}^{j}
$$

and power counting indicates that, in a perturbative expansion about flat space, it is a super-renomalizable model, with superficial degree of divergence $D=2-L$ where $L$ counts the number of loops [2]. Thus, viewing quantum mechanics as a particular QFT in one euclidean dimension one finds that possible divergences may arise at one- and twoloops. Therefore, just like in generic QFTs, one must define a regularization scheme with corresponding counterterms. Usually counterterms contain an infinte part, needed to cancel divergences, and a finite part, needed to match the renomalization conditions. In the present case the counterterms are finite if one includes the local terms arising from the general coordinate invariant path integral measure.

Three well-defined regularizations have been studied in the literature, all prompted by the effort of computing QFT trace anomalies with quantum mechanical path integrals [14, 15]. The latter extended to trace anomalies the quantum mechanical method used for chiral anomalies in [16-18]. In the case of chiral anomalies the presence of a worldline supersymmetry carries many simplifications. However, supersymmetry is not present in the trace anomaly case, and the corresponding quantum mechanical path integrals must be defined with great care to keep under control the full perturbative expansion.

To recall the various regularization schemes let us first notice that in configuration space the formally covariant measure can be related to a translational invariant measure by using ghost fields $a^{i}, b^{i}$ and $c^{i}$ à la Feddeev-Popov

$$
\mathcal{D} x=\prod_{0<t<\beta} d^{d} x(t) \sqrt{g(x(t))}=\prod_{0<t<\beta} d^{d} x(t) \int D a D b D c e^{-S_{g h}[x, a, b, c]}
$$

where

$$
S_{g h}[x, a, b, c]=\int_{0}^{\beta} d t \frac{1}{2} g_{i j}(x)\left(a^{i} a^{j}+b^{i} c^{j}\right)
$$


Considering $a^{i}$ bosonic variable and $b^{i}, c^{i}$ fermionic variables allows to reproduce the factor $\frac{g(x(t))}{\sqrt{g(x(t))}}=\sqrt{g(x(t))}$ in the measure. By $D x, D a, D b$ and $D c$ we indicate the translational invariant measure, useful for generating the perturbative expansion (e.g. $D x=$ $\prod_{0<t<\beta} d^{d} x(t)$, and so on). Thus, the path integral for the nonlinear sigma model in configuration space can be written as

$$
\int D x D a D b D c e^{-S[x, a, b, c]}
$$

with the full action taking the form

$$
S[x, a, b, c]=\int_{0}^{\beta} d t\left(\frac{1}{2} g_{i j}(x)\left(\dot{x}^{i} \dot{x}^{j}+a^{i} a^{j}+b^{i} c^{j}\right)+V_{C T}\right)
$$

and with $V_{C T}$ indicating the counterterm associated to the chosen regularization. To generate the amplitude $K\left(x, x^{\prime} ; \beta\right)$ the paths $x(t)$ must of course satisfy the boundary conditions $x(0)=x^{\prime}$ and $x(\beta)=x$.

The time slicing regularization (TS) in configuration space was studied in [19, 20], by deriving it from the phase space path integral, and studying carefully the continuum limit of the propagators together with the rules that must be used in evaluating their products. Indeed one may recall that the perturbative propagators are distributions: how to multiply them and their derivatives together is the problem one faces in regulating the perturbative expansion. This regularization inherits the counterterm $V_{\mathrm{TS}}$ in (2.9).

Mode regularization (MR) was employed in curved space already in $[14,15]$. The complete counterterm was identified in [21] to address some mismatches originally found between TS and MR. With the correct counterterm

$$
V_{\mathrm{MR}}=-\frac{1}{8} R-\frac{1}{24} g_{i j} g^{k l} g^{m n} \Gamma_{k m}^{i} \Gamma_{l n}^{j}
$$

those mismatches disappeared. The rules how to define the products of distributions in this regularization scheme follows from expanding the quantum fluctuations in a Fourier series truncated by a cut-off, which eventually is removed to reach the continuum limit. Including the vertices originating from the counterterm produces the covariant final answer.

Finally, dimensional regularization (DR) was introduced in the quantum mechanical context in [22-24]. It needs the counterterm

$$
V_{\mathrm{DR}}=-\frac{1}{8} R
$$

which has the useful property of being covariant.

All these regularizations have been extensively tested and compared, see e.g. [25, 26]. Extensions to supersymmetric models have been recently discussed again in [27], where the counterterms in all the previous regularization schemes were identified for the supersymmetric nonlinear sigma model with $N$ supersymmetries at arbitrary $N$. Additional details on the various regularization schemes may be found in the book [2].

The case of trace anomalies provided a precise observable on which to test and verify the construction of the quantum mechanical path integrals in curved spaces, clearing the 
somewhat confusing status of the subject present in previous literature. With this tool at hand, more general applications of the path integral were possible, in particular in the first quantized approach to quantum fields [28] coupled to gravitational backgrounds, such as the worldline description of fields of spin $0,1 / 2$ and 1 coupled to gravity [29-32], the analysis of amplitudes in Einstein-Maxwell theory [33-36], the study of photon-graviton conversion in strong magnetic fields [37, 38], the description of higher spin fields in first quantization [39], as well as worldline approaches to perturbative quantum gravity [40].

\section{A linear sigma model}

In the previous section we have reviewed the quantum mechanical path integral for a nonlinear sigma model, that describes a particle moving in a curved space by using arbitrary coordinates. In this section we wish to take up in a critical way an old proposal, put forward by Guven in [3], of constructing the path integral in curved space by using Riemann normal coordinates. The proposal assumes that in Riemann coordinates an auxiliary flat metric can be used in the kinetic term, while an effective potential reproduces the effects of the curved space. This construction aims at transforming the original nonlinear sigma model into a linear one. If correct, it carries several simplifications, making perturbative calculations simpler and more efficient. It may also improve its use in the worldline applications mentioned earlier.

Thus, let us review the considerations put forward in [3]. First of all it is convenient to consider the transition amplitude as a bidensity by defining

$$
\bar{K}\left(x, x^{\prime}, \beta\right)=g^{\frac{1}{4}}(x) K\left(x, x^{\prime}, \beta\right) g^{\frac{1}{4}}\left(x^{\prime}\right)
$$

so that, from (2.6), $\bar{K}$ is seen to satisfy the equation

$$
-\frac{\partial}{\partial \beta} \bar{K}\left(x, x^{\prime} ; \beta\right)=-\frac{1}{2} g^{\frac{1}{4}}(x) \nabla_{x}^{2} g^{-\frac{1}{4}}(x) \bar{K}\left(x, x^{\prime} ; \beta\right)
$$

with boundary condition

$$
\bar{K}\left(x, x^{\prime} ; 0\right)=\delta^{(d)}\left(x-x^{\prime}\right)
$$

where $\nabla_{x}^{2}$ is the scalar laplacian $\nabla^{2}=\frac{1}{\sqrt{g}} \partial_{i} \sqrt{g} g^{i j} \partial_{j}$ acting on the $x$ coordinates. The differential operator appearing on the right hand side of eq. (3.2) can be rewritten through a direct computation as

$$
-\frac{1}{2} g^{\frac{1}{4}} \nabla^{2} g^{-\frac{1}{4}}=-\frac{1}{2} \partial_{i} g^{i j} \partial_{j}+V_{\text {eff }}
$$

where derivatives act through and with the effective potential given by

$$
V_{\text {eff }}=-\frac{1}{2} g^{\frac{1}{4}} \nabla^{2} g^{-\frac{1}{4}}=-\frac{1}{2} g^{-\frac{1}{4}} \partial_{i} \sqrt{g} g^{i j} \partial_{j} g^{-\frac{1}{4}}
$$

where all derivatives now stop after acting on the last function. At this stage, one may use Riemann normal coordinates (see [41, 42], and also [43, 44] for their application to nonlinear sigma models). It was claimed in [6] that the Lorentz invariance (rotational 
invariance in euclidean conventions) of the momentum-space representation of $\bar{K}$ written in Riemann normal coordinates implies that the $g^{i j}$ in the $\partial_{i} g^{i j} \partial_{j}$ operator of (3.4) can be replaced by the constant $\delta^{i j}$. Indeed, in the momentum-space representation of $\bar{K}$ previously studied in ref. [5] by using Riemann normal coordinates, it was found that in an adiabatic expansion of $\bar{K}$ the first few terms depended on certain scalar functions, which were functions of $\delta_{i j} x^{i} x^{j}$ only (see also the book [7]). However it is not obvious why such a property should hold to all orders. In a curved space Lorentz invariance obviously cannot hold, for example scalar terms proportional to $R_{i j} x^{i} x^{j}$ may also arise (by $R_{i j}$ we consider the Ricci tensor evaluated at the origin of the Riemann coordinates, and by $x^{i}$ the Riemann normal coordinates themselves). Guven in [3] claimed however that in Riemann normal coordinates eq. (3.4) simplifies to

$$
-\frac{1}{2} g^{\frac{1}{4}} \nabla^{2} g^{-\frac{1}{4}}=-\frac{1}{2} \delta^{i j} \partial_{i} \partial_{j}+V_{\text {eff }}
$$

while referring to [45] for a proof. Thus he was led to consider the euclidean Schroedinger equation

$$
-\frac{\partial}{\partial \beta} \bar{K}\left(x, x^{\prime} \beta\right)=\left(-\frac{1}{2} \delta^{i j} \partial_{i} \partial_{j}+V_{\mathrm{eff}}(x)\right) \bar{K}\left(x, x^{\prime} ; \beta\right)
$$

that can be solved by a standard path integral for a linear sigma model

$$
\bar{K}\left(x, x^{\prime} ; \beta\right) \sim \int_{x(0)=x^{\prime}}^{x(\beta)=x} D x e^{-S[x]}, \quad S[x]=\int_{0}^{\beta} d t\left(\frac{1}{2} \delta_{i j} \dot{x}^{i} \dot{x}^{j}+V_{\text {eff }}(x)\right) .
$$

However again, in reviewing this construction, we have not been able to find the proof of (3.6) in [45], which does not contain such statements. Also the effective potential used in [3] does not coincide with the one written in eq. (3.5) (even taking care of the different conventions used). In any case, it is the potential in (3.5) that might have a chance of working.

Given this state of understanding, we still find the conjecture that "the path integral in curved space can be reduced in Riemann normal coordinates to that of a linear sigma model" to be rather appealing. Also, the reasonings leading to (3.6) has a better chance of working if one considers maximally symmetric spaces, where Lorentz (or rotational) symmetry can indeed be implemented in a suitable sense. This is indeed the case, and we prove in appendix A that the bidensity (3.1), on a $d$-dimensional maximally symmetric space described by Riemann normal coordinates satisfies the heat equation with the flat operator (3.4). Thus, in the next sections, we proceed in testing explicitely the path integral construction on spaces of maximal symmetry.

\section{Path integral on maximally symmetric spaces}

We wish to test the path integral in Riemann normal coordinates using the linear sigma model of eq. (3.8) and considering maximally symmetric spaces. In particular, we wish to compare it with the path integral calculation done with the nonlinear sigma model and Riemann normal coordinates in [4]. The observable computed there was the transition 
amplitude at coinciding points $K(x, x, \beta)$. In the present analysis we use the same notations of ref. [4], except for a change in sign in the Ricci tensors, so to have a positive Ricci scalar on spheres.

On maximally symmetric spaces the Riemann tensor is related to the metric tensor by

$$
R_{m n a b}=M^{2}\left(g_{m a} g_{n b}-g_{m b} g_{n a}\right)
$$

where $M^{2}$ is a constant that can be either positive, negative, or vanishing (flat space). The Ricci tensors are then defined by

$$
\begin{aligned}
R_{m n} & =R_{a m}{ }^{a}{ }_{n}=M^{2}(d-1) g_{m n} \\
R & =R_{m}{ }^{m}=M^{2}(d-1) d
\end{aligned}
$$

so that the constant $M^{2}$ is related to the constant Ricci scalar $R$ by

$$
M^{2}=\frac{R}{(d-1) d}
$$

which is positive on a sphere. We want to use Riemann normal coordinates. The expansion of the metric in normal coordinates around a point (called the origin) is obtained by standard methods and reads

$$
g_{m n}(x)=\delta_{m n}+\left(\delta_{m n}-\hat{x}_{m} \hat{x}_{n}\right)\left(-\frac{1}{3}(M x)^{2}+\frac{32}{6 !}(M x)^{4}-\frac{16}{7 !}(M x)^{6}+\cdots\right)
$$

where $x^{m}$ denote now Riemann normal coordinates and

$$
x=\sqrt{\vec{x}^{2}}, \quad \hat{x}^{m}=\frac{x^{m}}{x} .
$$

One may compute all terms of the series recursively, and sum the series to get [4]

$$
\begin{aligned}
g_{m n}(x) & =\delta_{m n}+P_{m n} \sum_{n=1}^{\infty} \frac{2(-1)^{n}}{(2 n+2) !}(2 M x)^{2 n} \\
& =\delta_{m n}+P_{m n} \frac{1-2(M x)^{2}-\cos (2 M x)}{2(M x)^{2}}
\end{aligned}
$$

where the projector $P_{m n}$ is defined by

$$
P_{m n}=\delta_{m n}-\hat{x}_{m} \hat{x}_{n} .
$$

Defining the auxiliary functions

$$
f(x)=\frac{1-2(M x)^{2}-\cos (2 M x)}{2(M x)^{2}}, \quad h(x)=-\frac{f(x)}{1+f(x)}
$$

allows to write the metric, its inverse, and the metric determinant in Riemann normal coordinates as

$$
\begin{aligned}
g_{m n}(x) & =\delta_{m n}+f(x) P_{m n} \\
g^{m n}(x) & =\delta^{m n}+h(x) P^{m n} \\
g(x) & =(1+f(x))^{d-1}
\end{aligned}
$$


where, on the right hand side of these formulae, indices are raised and lowered with the flat metric $\delta_{m n}$.

We are now ready to consider the linear sigma model (3.8). We wish to evaluate the transition amplitude at coinciding points $x=x^{\prime}=0$ (taken to be the origin of the Riemann coordinates) in a perturbative expansion in terms of the propagation time $\beta$. To control the $\beta$ expansion it is useful to rescale the time $t \rightarrow \tau=\frac{t}{\beta}$ so that $\tau \in[0,1]$ and the action takes the form

$$
S[x]=\int_{0}^{1} d \tau\left(\frac{1}{2 \beta} \delta_{i j} \dot{x}^{i} \dot{x}^{j}+\beta V_{\mathrm{eff}}(x)\right)
$$

The leading term for $\beta \rightarrow 0$ is just the free particle which is exactly solvable. It is notationally convenient to set $M=1$, as $M$ can be reintroduced by dimensional analysis. Now we must compute the potential $V_{\text {eff }}(x)$. Using eqs. (4.8) and (4.9), from (3.5) we find

$$
V_{\mathrm{eff}}(x)=\frac{(d-1)}{8}\left[\frac{(d-5)}{4}\left(\frac{f^{\prime}}{1+f}\right)^{2}+\frac{1}{1+f}\left(\frac{(d-1)}{x} f^{\prime}+f^{\prime \prime}\right)\right]
$$

which is evaluated to

$$
V_{\text {eff }}(x)=\frac{d-d^{2}}{12}+\frac{(d-1)(d-3)}{48} \frac{\left(5 x^{2}-3+\left(x^{2}+3\right) \cos (2 x)\right)}{x^{2} \sin ^{2}(x)}
$$

and which expands to

$$
\begin{aligned}
V_{\mathrm{eff}}(x)=\frac{d-d^{2}}{12}+(d-1)(d-3)( & \frac{x^{2}}{120}+\frac{x^{4}}{756}+\frac{x^{6}}{5400}+\frac{x^{8}}{41580}+ \\
& \left.+\frac{691 x^{10}}{232186500}+\frac{x^{12}}{2806650}+O\left(x^{14}\right)\right) .
\end{aligned}
$$

The perturbative expansion of the path integral is obtained by setting

$$
S[x]=S_{\text {free }}[x]+S_{\text {int }}[x]
$$

with

$$
S_{\text {free }}[x]=\frac{1}{\beta} \int_{0}^{1} d \tau \frac{1}{2} \delta_{i j} \dot{x}^{i} \dot{x}^{j}, \quad S_{\text {int }}[x]=\beta \int_{0}^{1} d \tau V_{\text {eff }}(x)
$$

so that eq. (3.1) reduces to ( $x=0$ is the Riemann normal coordinate of the origin)

$$
\bar{K}(0,0 ; \beta)=\frac{\left\langle e^{-S_{\text {int }}}\right\rangle}{(2 \pi \beta)^{\frac{d}{2}}}
$$

where $\langle\ldots\rangle$ denotes normalized correlation function with the free path integral. 
Using the free propagator and Wick contractions, we obtain the following perturbative answer (see appendix B for details)

$$
\begin{aligned}
& \bar{K}(0,0 ; \beta)=\frac{1}{(2 \pi \beta)^{\frac{d}{2}}} \exp \left[\frac{\beta R}{12}-\frac{(\beta R)^{2}}{6 !} \frac{(d-3)}{d(d-1)}-\frac{(\beta R)^{3}}{9 !} \frac{16(d-3)(d+2)}{d^{2}(d-1)^{2}}\right. \\
&-\frac{(\beta R)^{4}}{10 !} \frac{2(d-3)\left(d^{2}+20 d+15\right)}{d^{3}(d-1)^{3}} \\
&+\frac{(\beta R)^{5}}{11 !} \frac{8(d-3)(d+2)\left(d^{2}-12 d-9\right)}{3 d^{4}(d-1)^{4}} \\
&+ \\
&+\frac{(\beta R)^{6}}{13 !} \frac{8(d-3)\left(1623 d^{4}-716 d^{3}-65930 d^{2}-123572 d-60165\right)}{315 d^{5}(d-1)^{5}} \\
&+\left.O\left(\beta^{7}\right)\right]
\end{aligned}
$$

with the exponential that can be expanded to identify the first six heat kernel coefficients (also known as Seeley-DeWitt coefficients).

Amazingly, it compares successfully with eq. (16) of ref. [4] (taking into account that $\xi=0$ and that the sign of $R$ has been reversed). In that reference the calculation was performed up to order $(\beta R)^{3}$. In the present case those results are reproduced almost trivially, and in fact we have been able to push the calculation to higher orders. For arbitrary $d$ these higher orders are new, as far as we know. In the next section we will further test our coefficients at specific values of $d$. It is also amusing to note that the path integral result is exact on the 3 -sphere, as the effective potential $V_{\text {eff }}$ in eq. (4.12) becomes constant at $d=3$. This is as it should be, as the transition amplitude on $S^{3}$ is known exactly [46], thanks to the fact that $S^{3}$ coincides with the group manifold $\mathrm{SU}(2)$.

\section{Type-A trace anomaly of a scalar field}

A further test is to use our results to compute the type-A trace anomaly of a conformal scalar field. Trace anomalies characterize conformal field theories. They amount to the fact that the trace of the energy-momentum tensor for conformal fields, which vanishes at the classical level, acquires anomalous terms at the quantum level. These terms depend on the background geometry of the spacetime on which the conformal fields are coupled to, and they are captured by the appropriate Seeley-DeWitt coefficient sitting in the heat kernel expansion of the associated conformal operator, see [47] for example.

A simple way to obtain this relation is to view the trace anomaly as due to the QFT path integral measure, so that it is computed by the regulated Jacobian arising from the Weyl transformation of the QFT path integral measure [48]. For a scalar field the infinitesimal Weyl transformation $\delta_{\sigma} g_{m n}(x)=\sigma(x) g_{m n}(x)$, applied to the one-loop effective action, yields

$$
\int d^{d} x \sqrt{g} \sigma(x)\left\langle T^{m}{ }_{m}(x)\right\rangle=\lim _{\beta \rightarrow 0} \operatorname{Tr}\left\{\sigma e^{-\beta \mathcal{R}}\right\}
$$


where the consistent regulator $\mathcal{R}$, that appears in the exponent, is just the conformal operator associated to the scalar field, and reads

$$
\mathcal{R}=-\frac{1}{2} \nabla^{2}+\frac{\xi}{2} R
$$

with $\xi=\frac{d-2}{4(d-1)}$. It can be identified as the hamiltonian operator (2.3) for a non-relativistic particle in curved space. Therefore, one identifies the trace anomaly in terms of a particle path integral by

$$
\left\langle T^{m}{ }_{m}(x)\right\rangle=\lim _{\beta \rightarrow 0} K(x, x ; \beta)
$$

where it is understood that the limit picks up just the $\beta$-independent term - divergent terms are removed by QFT renormalization. This procedure selects the appropriate SeeleyDeWitt coefficient sitting in the expansion of $K(x, x ; \beta)$.

Trace anomalies have been classified as type-A, type-B and trivial anomalies in [49]. On conformally flat spaces the type-B and trivial anomalies vanish, so that only the type-A anomaly survives. It is proportional to the topological Euler density, and its coefficient enters the so-called $c$-theorem of 2 dimensions [50] and $a$-theorem of 4 dimensions [51] at fixed points. These theorems capture the irreversibility of the renormalization group flow in 2 and 4 dimensions. Their extension to arbitrary even dimensions has been conjectured, but not proven (see also [52] for a more general conjecture).

We are going to use the previous results on the sphere (a conformally flat space) to calculate the type-A trace anomaly for a scalar field in arbitrary dimensions up to $d=12$, which will serve as a further test on the linear sigma model approach of the previous section. Using the expansion obtained in the previous section, and choosing $x$ as the origin of the RNC coordinate system, we have by definition $g_{m n}(x)=\delta_{m n}$ in Riemann normal coordinates, and

$$
\left\langle T^{m}{ }_{m}(x)\right\rangle=\lim _{\beta \rightarrow 0} \bar{K}(x, x ; \beta)
$$

so that expanding (4.17) (recall that there $x=0$ indicates the origin of the RNC), and picking the $\beta^{0}$ term in the chosen dimension $d$, we obtain the trace anomalies for a conformal scalar field in $d$ dimensions reported in table 1 , where the second form is written in terms of $a^{2}=\frac{1}{M^{2}}=\frac{d(d-1)}{R}$ to directly compare with the results tabulated in [53].

The comparison is successful, except at $d=12$, where our respective coefficients differ by a number of the order of $10^{-13}$. Our result is correct as using the zeta function approach employed in $[53,54]$ we have been able to reproduce our findings. ${ }^{3}$ It compares well also with a nice formula obtained in [55] using AdS/CFT arguments, and confirmed later in $[56,57]$, for evaluating the type-A trace anomaly of conformal scalar operators. The direct evaluation of that formula produces, modulo different normalizations, the correct numerator 133787 of the anomaly at $d=12$.

\footnotetext{
${ }^{3}$ The mismatch could perhaps have happened due to some inappropriate rounding of the exact number, occasionally introduced by calculators. We thank Zura Kakushadze for having pointed out such a possibility to us.
} 


\begin{tabular}{|l|l|l|}
\hline$d$ & $\left\langle T^{\mu}{ }_{\mu}\right\rangle$ & $\left\langle T^{\mu}{ }_{\mu}\right\rangle$ \\
\hline 2 & $\frac{R}{24 \pi}$ & $\frac{1}{12 \pi a^{2}}$ \\
\hline 4 & $-\frac{R^{2}}{34560 \pi^{2}}$ & $-\frac{1}{240 \pi^{2} a^{4}}$ \\
\hline 6 & $\frac{R^{3}}{21772800 \pi^{3}}$ & $\frac{5}{4032 \pi^{3} a^{6}}$ \\
\hline 8 & $-\frac{23 R^{4}}{339880181760 \pi^{4}}$ & $-\frac{23}{34560 \pi^{4} a^{8}}$ \\
\hline 10 & $\frac{263 R^{5}}{2993075712000000 \pi^{5}}$ & $\frac{263}{506880 \pi^{5} a^{10}}$ \\
\hline 12 & $-\frac{133787 R^{6}}{1330910037208675123200 \pi^{6}}$ & $-\frac{133787}{251596800 \pi^{6} a^{12}}$ \\
\hline
\end{tabular}

Table 1. The type-A trace anomaly of a scalar field.

\section{Conclusions}

We have tested a method of computing the path integral for a particle in curved spaces in Riemann normal coordinates that employs a linear sigma model action with an additional scalar effective potential. This method was proposed by Guven in [3], but with assumptions whose proof were not given. We have checked the method by restricting it to maximally symmetric geometries, and found that indeed it reproduces correct results in a quite efficient way. In particular, we have used it to obtain the first six Seeley-DeWitt coefficients at coinciding points for the $d$-dimensional sphere (more generally, for maximally symmetric spaces), and computed the type-A trace anomaly for a scalar field up to $d=12$. This helped us also to correct a wrong value for the trace anomaly of a scalar field in twelve dimensions reported in ref. [53]. The success of the simplified path integral on maximally symmetric spaces has led us to search for a simple proof of its validity, which we have found and reported in appendix A.

It would be interesting to extend the present method to supersymmetric nonlinear sigma models, so to consider fields of spin $1 / 2$ and 1 , if not higher, in worldline applications, or to consider curved spaces with boundaries, following the path integral treatment of refs. $[58,59]$ which dealt with flat space only.

As for arbitrary geometries, we cannot say much at this stage. If a proof of the crucial relation used in constructing the path integral cannot be produced, one may still test it by a perturbative computation at sufficiently high order. We wish to be able to report on this subject in a near future. 


\section{Acknowledgments}

We would like to thank Andrej Barvinski and Christian Schubert for useful discussions.

\section{A A simple proof in maximally symmetric spaces}

Here we give a simple proof that the bidensity (3.1) satisfies the heat equation

$$
-\frac{\partial}{\partial \beta} \bar{K}\left(x, x^{\prime} \beta\right)=\left(-\frac{1}{2} \delta^{i j} \partial_{i} \partial_{j}+V_{\mathrm{eff}}(x)\right) \bar{K}\left(x, x^{\prime} ; \beta\right)
$$

in a maximally symmetric space described by Riemann normal coordinates. For this to be true we must show that the "curved" differential operator (3.4) acts on (3.1) identically as the "flat" operator (3.6), i.e.

$$
\left(\partial_{i} g^{i j} \partial_{j}-\delta^{i j} \partial_{i} \partial_{j}\right) \bar{K}\left(x, x^{\prime} ; \beta\right)=0 .
$$

Taking $x^{\prime}=0$ as the origin of the Riemann normal coordinates, and using (4.7) and (4.9), the left hand side of (A.2) reduces to

$$
\left[h(x) P^{i j}(x) \partial_{i} \partial_{j}+\partial_{i}\left(h(x) P^{i j}(x)\right) \partial_{j}\right] \bar{K}(x, 0 ; \beta) .
$$

In maximally symmetric spaces, all curvature tensors are given algebraically in terms of the metric and of the constant scalar curvature $R$, see eqs. (4.1)-(4.3), so that by symmetry arguments the bidensity $\bar{K}(x, 0 ; \beta)$ can only depend on the coordinates through the "scalar" function $x^{2}=\delta_{i j} x^{i} x^{j}$. Therefore, using the orthogonality condition $P^{i j} x_{j}=0$, one gets

$$
\partial_{i}\left(h(x) P^{i j}(x)\right) \partial_{j} \bar{K}(x, 0 ; \beta)=-2(d-1) h(x) \frac{\partial}{\partial x^{2}} \bar{K}(x, 0 ; \beta)
$$

and

$$
h(x) P^{i j}(x) \partial_{i} \partial_{j} \bar{K}(x, 0 ; \beta)=2 h(x) \delta_{i j} P^{i j}(x) \frac{\partial}{\partial x^{2}} \bar{K}(x, 0 ; \beta)=2(d-1) h(x) \frac{\partial}{\partial x^{2}} \bar{K}(x, 0 ; \beta) .
$$

Therefore, (A.2) is proven. Casting (A.1) in the form of a path integral is now immediate.

\section{B Computational details}

The free propagator for $x^{i}(\tau)$ vanishing at $\tau=0$ and $\tau=1$ is obtained from (4.15) and reads

$$
\left\langle x^{i}(\tau) x^{j}(\sigma)\right\rangle=-\beta \delta^{i j} \Delta(\tau, \sigma)
$$

with

$$
\begin{aligned}
\Delta(\tau, \sigma) & =(\tau-1) \sigma \theta(\tau-\sigma)+(\sigma-1) \tau \theta(\sigma-\tau) \\
& =\frac{1}{2}|\tau-\sigma|-\frac{1}{2}(\tau+\sigma)+\tau \sigma
\end{aligned}
$$

where $\theta(x)$ is the Heaviside step function with $\theta(0)=\frac{1}{2}$. 
The perturbative expansion is obtained from (4.16). In expanding the exponential with $S_{\text {int }}$ it is useful to define

$$
S_{\text {int }}=\sum_{m=0}^{\infty} S_{2 m}
$$

where $S_{2 m}$ is the term containing the power $\left(x^{2}\right)^{m}$, with $x^{2}=\vec{x}^{2}=x^{i} x_{i}$. For simplicity we denote them by

$$
S_{2 m}=\beta k_{2 m} \int_{0}^{1} d \tau\left(x^{2}\right)^{m}
$$

where the numerical coefficients $k_{2 m}$ are read off from (4.13). It is sufficient to compute the connected correlation functions, denoted by $\langle\ldots\rangle_{c}$, and express $(4.16)$ as

$$
\begin{aligned}
\bar{K}(0,0 ; \beta)= & \frac{e^{-S_{0}}}{(2 \pi \beta)^{\frac{d}{2}}} \exp \left[-\left\langle S_{2}\right\rangle-\left\langle S_{4}\right\rangle-\left\langle S_{6}\right\rangle-\left\langle S_{8}\right\rangle-\left\langle S_{10}\right\rangle\right. \\
& \left.+\frac{1}{2}\left\langle S_{2}^{2}\right\rangle_{c}+\left\langle S_{2} S_{4}\right\rangle_{c}+\frac{1}{2}\left\langle S_{4}^{2}\right\rangle_{c}+\left\langle S_{2} S_{6}\right\rangle_{c}-\frac{1}{3 !}\left\langle S_{2}^{3}\right\rangle_{c}+O\left(\beta^{7}\right)\right]
\end{aligned}
$$

where we have kept terms contributing up to order $\beta^{6}$ only. Using Wick contractions we find the following result

$$
\begin{aligned}
\bar{K}(0,0 ; \beta)= & \frac{1}{(2 \pi \beta)^{\frac{d}{2}}} \exp \left[\beta \frac{d(d-1)}{12}-(d-1)(d-3)\left(\beta^{2} \frac{d}{720}+\beta^{3} \frac{d(d+2)}{22680}\right.\right. \\
& +\beta^{4} \frac{d\left(d^{2}+20 d+15\right)}{1814400}-\beta^{5} \frac{d(d+2)\left(d^{2}-12 d-9\right)}{14968800} \\
& \left.\left.-\beta^{6} \frac{d\left(1623 d^{4}-716 d^{3}-65930 d^{2}-123572 d-60165\right)}{245188944000}\right)+O\left(\beta^{7}\right)\right]
\end{aligned}
$$

where the intermediate results that we have summed here above are as follows (using the abbreviation $\left.\Delta\left(\tau_{1}, \tau_{2}\right) \equiv \Delta_{12}\right)$

Order $\boldsymbol{\beta}$. There is only a constant term that does not require any Wick contraction

$$
-S_{0}=\beta \frac{d(d-1)}{12}
$$

Order $\beta^{2}$.

$$
-\left\langle S_{2}\right\rangle=\beta^{2} k_{2} d \underbrace{\int_{0}^{1} d \tau_{1} \Delta_{11}}_{-\frac{1}{6}}
$$

Order $\beta^{3}$.

$$
-\left\langle S_{4}\right\rangle=-\beta^{3} k_{4} d(d+2) \underbrace{\int_{0}^{1} d \tau_{1} \Delta_{11}^{2}}_{\frac{1}{30}}
$$


Order $\beta^{4}$.

$$
\begin{aligned}
-\left\langle S_{6}\right\rangle & =\beta^{4} k_{6} \underbrace{\left(d^{3}+6 d^{2}+8 d\right)}_{d(d+2)(d+4)} \underbrace{\int_{0}^{1} d \tau_{1} \Delta_{11}^{3}}_{-\frac{1}{140}} \\
\frac{1}{2}\left\langle S_{2}^{2}\right\rangle_{c} & =\beta^{4} k_{2}^{2} d \underbrace{\int_{0}^{1} d \tau_{1} \int_{0}^{1} d \tau_{2} \Delta_{12}^{2}}_{\frac{1}{90}}
\end{aligned}
$$

Order $\beta^{5}$.

$$
\begin{aligned}
-\left\langle S_{8}\right\rangle & =-\beta^{5} k_{8} \underbrace{\left(d^{4}+12 d^{3}+(12+32) d^{2}+48 d\right)}_{d(d+2)(d+4)(d+6)} \underbrace{\int_{0}^{1} d \tau_{1} \Delta_{11}^{4}}_{\frac{1}{630}} \\
\left\langle S_{2} S_{4}\right\rangle_{c} & =-\beta^{5} k_{2} k_{4}\left(4 d^{2}+8 d\right) \underbrace{\int_{0}^{1} d \tau_{1} \int_{0}^{1} d \tau_{2} \Delta_{12}^{2} \Delta_{22}}_{-\frac{1}{420}}
\end{aligned}
$$

Order $\beta^{6}$.

$$
\begin{aligned}
& -\left\langle S_{10}\right\rangle=\beta^{6} k_{10} \underbrace{\left(d^{5}+20 d^{4}+(80+60) d^{3}+(240+160) d^{2}+384 d\right)}_{d(d+2)(d+4)(d+6)(d+8)} \underbrace{\int_{0}^{1} d \tau_{1} \Delta_{11}^{5}}_{-\frac{1}{2772}} \\
& -\frac{1}{3 !}\left\langle S_{2}^{3}\right\rangle_{c}=\frac{\beta^{6}}{3 !} k_{2}^{3} 8 d \underbrace{\int_{0}^{1} d \tau_{1} \int_{0}^{1} d \tau_{2} \int_{0}^{1} d \tau_{3} \Delta_{12} \Delta_{23} \Delta_{31}}_{-\frac{1}{945}} \\
& \left\langle S_{2} S_{6}\right\rangle_{c}=\beta^{6} k_{2} k_{6} 6 d\left(d^{2}+6 d+8\right) \underbrace{\int_{0}^{1} d \tau_{1} \int_{0}^{1} d \tau_{2} \Delta_{12}^{2} \Delta_{22}^{2}}_{\frac{1}{1890}} \\
& \frac{1}{2}\left\langle S_{4}^{2}\right\rangle_{c}=\frac{\beta^{6}}{2} k_{4}^{2}(8 d(d+2) \underbrace{\int_{0}^{1} d \tau_{1} \int_{0}^{1} d \tau_{2} \Delta_{12}^{4}}_{\frac{1}{3150}} \\
& +8 d\left(d^{2}+4 d+4\right) \underbrace{\int_{0}^{1} d \tau_{1} \int_{0}^{1} d \tau_{2} \Delta_{11} \Delta_{12}^{2} \Delta_{22}}_{\frac{13}{25200}}) .
\end{aligned}
$$

Open Access. This article is distributed under the terms of the Creative Commons Attribution License (CC-BY 4.0), which permits any use, distribution and reproduction in any medium, provided the original author(s) and source are credited. 


\section{References}

[1] R.P. Feynman, Space-time approach to nonrelativistic quantum mechanics, Rev. Mod. Phys. 20 (1948) 367 [InSPIRE].

[2] F. Bastianelli and P. van Nieuwenhuizen, Path integrals and anomalies in curved space, Cambridge University Press, Cambridge U.K., (2006) [INSPIRE].

[3] J. Guven, Calculating the effective action for a selfinteracting scalar quantum field theory in a curved background space-time, Phys. Rev. D 37 (1988) 2182 [INSPIRE].

[4] F. Bastianelli and N.D. Hari Dass, Simplified method for trace anomaly calculations in $D=6$ and $d \leq 6$, Phys. Rev. D 64 (2001) 047701 [hep-th/0104234] [InSPIRE].

[5] T.S. Bunch and L. Parker, Feynman propagator in curved space-time: a momentum space representation, Phys. Rev. D 20 (1979) 2499 [InSPIRE].

[6] B.L. Hu and D.J. O'Connor, Effective Lagrangian for $\lambda \phi^{4}$ theory in curved space-time with varying background fields: quasilocal approximation, Phys. Rev. D 30 (1984) 743 [InSPIRE].

[7] L.E. Parker and D. Toms, Quantum field theory in curved spacetime: quantized fields and gravity, Cambridge University Press, Cambridge U.K., (2009) [InSPIRE].

[8] B.S. DeWitt, Dynamical theory in curved spaces. 1. A review of the classical and quantum action principles, Rev. Mod. Phys. 29 (1957) 377 [InSPIRE].

[9] F.A. Berezin, Non-Wiener continual integrals, Theor. Math. Phys. 6 (1971) 141 [Teor. Mat. Fiz. 6 (1971) 194] [INSPIRE].

[10] M.M. Mizrahi, The Weyl correspondence and path integrals, J. Math. Phys. 16 (1975) 2201 [INSPIRE].

[11] M.-A. Sato, Operator ordering and perturbation expansion in the path integration formalism, Prog. Theor. Phys. 58 (1977) 1262 [INSPIRE].

[12] J.-L. Gervais and A. Jevicki, Point canonical transformations in path integral, Nucl. Phys. B 110 (1976) 93 [InSPIRE].

[13] B. Sakita, Quantum theory of many variable systems and fields, World Scientific, Singapore, (1985) [INSPIRE].

[14] F. Bastianelli, The path integral for a particle in curved spaces and Weyl anomalies, Nucl. Phys. B 376 (1992) 113 [hep-th/9112035] [INSPIRE].

[15] F. Bastianelli and P. van Nieuwenhuizen, Trace anomalies from quantum mechanics, Nucl. Phys. B 389 (1993) 53 [hep-th/9208059] [INSPIRE].

[16] L. Álvarez-Gaumé, Supersymmetry and the Atiyah-Singer index theorem, Commun. Math. Phys. 90 (1983) 161 [InSPIRE].

[17] L. Álvarez-Gaumé and E. Witten, Gravitational anomalies, Nucl. Phys. B 234 (1984) 269 [INSPIRE].

[18] D. Friedan and P. Windey, Supersymmetric derivation of the Atiyah-Singer index and the chiral anomaly, Nucl. Phys. B 235 (1984) 395 [inSPIRE].

[19] J. De Boer, B. Peeters, K. Skenderis and P. Van Nieuwenhuizen, Loop calculations in quantum mechanical nonlinear $\sigma$-models, Nucl. Phys. B 446 (1995) 211 [hep-th/9504097] [INSPIRE].

[20] J. de Boer, B. Peeters, K. Skenderis and P. van Nieuwenhuizen, Loop calculations in quantum mechanical nonlinear $\sigma$-models $\sigma$-models with fermions and applications to anomalies, Nucl. Phys. B 459 (1996) 631 [hep-th/9509158] [INSPIRE]. 
[21] F. Bastianelli, K. Schalm and P. van Nieuwenhuizen, Mode regularization, time slicing, Weyl ordering and phase space path integrals for quantum mechanical nonlinear $\sigma$-models, Phys. Rev. D 58 (1998) 044002 [hep-th/9801105] [INSPIRE].

[22] H. Kleinert and A. Chervyakov, Reparametrization invariance of path integrals, Phys. Lett. B 464 (1999) 257 [hep-th/9906156] [INSPIRE].

[23] F. Bastianelli, O. Corradini and P. van Nieuwenhuizen, Dimensional regularization of the path integral in curved space on an infinite time interval, Phys. Lett. B 490 (2000) 154 [hep-th/0007105] [INSPIRE].

[24] F. Bastianelli, O. Corradini and P. van Nieuwenhuizen, Dimensional regularization of nonlinear $\sigma$-models on a finite time interval, Phys. Lett. B 494 (2000) 161 [hep-th/0008045] [INSPIRE].

[25] F. Bastianelli and O. Corradini, On mode regularization of the configuration space path integral in curved space, Phys. Rev. D 60 (1999) 044014 [hep-th/9810119] [INSPIRE].

[26] F. Bastianelli and O. Corradini, 6D trace anomalies from quantum mechanical path integrals, Phys. Rev. D 63 (2001) 065005 [hep-th/0010118] [INSPIRE].

[27] F. Bastianelli, R. Bonezzi, O. Corradini and E. Latini, Extended SUSY quantum mechanics: transition amplitudes and path integrals, JHEP 06 (2011) 023 [arXiv:1103.3993] [INSPIRE].

[28] C. Schubert, Perturbative quantum field theory in the string inspired formalism, Phys. Rept. 355 (2001) 73 [hep-th/0101036] [inSPIRE].

[29] F. Bastianelli and A. Zirotti, Worldline formalism in a gravitational background, Nucl. Phys. B 642 (2002) 372 [hep-th/0205182] [InSPIRE].

[30] F. Bastianelli, O. Corradini and A. Zirotti, Dimensional regularization for $N=1$ supersymmetric $\sigma$-models and the worldline formalism, Phys. Rev. D 67 (2003) 104009 [hep-th/0211134] [INSPIRE].

[31] F. Bastianelli, P. Benincasa and S. Giombi, Worldline approach to vector and antisymmetric tensor fields, JHEP 04 (2005) 010 [hep-th/0503155] [INSPIRE].

[32] F. Bastianelli, P. Benincasa and S. Giombi, Worldline approach to vector and antisymmetric tensor fields. II, JHEP 10 (2005) 114 [hep-th/0510010] [INSPIRE].

[33] T.J. Hollowood and G.M. Shore, The refractive index of curved spacetime: the fate of causality in QED, Nucl. Phys. B 795 (2008) 138 [arXiv:0707.2303] [INSPIRE].

[34] F. Bastianelli, J.M. Dávila and C. Schubert, Gravitational corrections to the Euler-Heisenberg Lagrangian, JHEP 03 (2009) 086 [arXiv:0812.4849] [INSPIRE].

[35] J.M. Dávila and C. Schubert, Effective action for Einstein-Maxwell theory at order $R F^{4}$, Class. Quant. Grav. 27 (2010) 075007 [arXiv: 0912 . 2384] [INSPIRE].

[36] F. Bastianelli, O. Corradini, J.M. Dávila and C. Schubert, On the low-energy limit of one-loop photon-graviton amplitudes, Phys. Lett. B 716 (2012) 345 [arXiv:1202.4502] [INSPIRE].

[37] F. Bastianelli and C. Schubert, One loop photon-graviton mixing in an electromagnetic field: part 1, JHEP 02 (2005) 069 [gr-qc/0412095] [INSPIRE].

[38] F. Bastianelli, U. Nucamendi, C. Schubert and V.M. Villanueva, One loop photon-graviton mixing in an electromagnetic field: part 2, JHEP 11 (2007) 099 [arXiv:0710.5572] [INSPIRE]. 
[39] F. Bastianelli, R. Bonezzi, O. Corradini and E. Latini, Effective action for higher spin fields on (A)dS backgrounds, JHEP 12 (2012) 113 [arXiv:1210.4649] [INSPIRE].

[40] F. Bastianelli and R. Bonezzi, One-loop quantum gravity from a worldline viewpoint, JHEP 07 (2013) 016 [arXiv: 1304.7135] [INSPIRE].

[41] L.P. Eisenhart, Riemannian geometry, Princeton University Press, Princeton U.S.A., (1965).

[42] A.Z. Petrov, Einstein spaces, Pergamon Press, Oxford U.K., (1969).

[43] L. Álvarez-Gaumé, D.Z. Freedman and S. Mukhi, The background field method and the ultraviolet structure of the supersymmetric nonlinear $\sigma$-model, Annals Phys. 134 (1981) 85 [INSPIRE].

[44] P.S. Howe, G. Papadopoulos and K.S. Stelle, The background field method and the nonlinear $\sigma$ model, Nucl. Phys. B 296 (1988) 26 [InSPIRE].

[45] G. Vilkovisky, The gospel according to DeWitt, in Quantum theory of gravity, S.M. Christensen ed., Adam Hilger Ltd., Bristol U.K., (1984), pg. 169 [InSPIRE].

[46] L. Schulman, A path integral for spin, Phys. Rev. 176 (1968) 1558 [INSPIRE].

[47] M.J. Duff, Twenty years of the Weyl anomaly, Class. Quant. Grav. 11 (1994) 1387 [hep-th/9308075] [INSPIRE].

[48] K. Fujikawa, Comment on chiral and conformal anomalies, Phys. Rev. Lett. 44 (1980) 1733 [INSPIRE].

[49] S. Deser and A. Schwimmer, Geometric classification of conformal anomalies in arbitrary dimensions, Phys. Lett. B 309 (1993) 279 [hep-th/9302047] [INSPIRE].

[50] A.B. Zamolodchikov, Irreversibility of the flux of the renormalization group in a $2 D$ field theory, JETP Lett. 43 (1986) 730 [Pisma Zh. Eksp. Teor. Fiz. 43 (1986) 565] [inSPIRE].

[51] Z. Komargodski and A. Schwimmer, On renormalization group flows in four dimensions, JHEP 12 (2011) 099 [arXiv: 1107.3987] [INSPIRE].

[52] S. Giombi and I.R. Klebanov, Interpolating between a and F, JHEP 03 (2015) 117 [arXiv: 1409.1937] [INSPIRE].

[53] E.J. Copeland and D.J. Toms, The conformal anomaly in higher dimensions, Class. Quant. Grav. 3 (1986) 431 [inSPIRE].

[54] A. Cappelli and G. D'Appollonio, On the trace anomaly as a measure of degrees of freedom, Phys. Lett. B 487 (2000) 87 [hep-th/0005115] [INSPIRE].

[55] D.E. Diaz, Polyakov formulas for GJMS operators from AdS/CFT, JHEP 07 (2008) 103 [arXiv: 0803.0571] [INSPIRE].

[56] J.S. Dowker, Entanglement entropy for even spheres, arXiv:1009.3854 [INSPIRE].

[57] J.S. Dowker, Determinants and conformal anomalies of GJMS operators on spheres, J. Phys. A 44 (2011) 115402 [arXiv:1010.0566] [INSPIRE].

[58] F. Bastianelli, O. Corradini and P.A.G. Pisani, Worldline approach to quantum field theories on flat manifolds with boundaries, JHEP 02 (2007) 059 [hep-th/0612236] [INSPIRE].

[59] F. Bastianelli, O. Corradini, P.A.G. Pisani and C. Schubert, Scalar heat kernel with boundary in the worldline formalism, JHEP 10 (2008) 095 [arXiv:0809.0652] [INSPIRE]. 